\title{
Confianza institucional y violencia estructural en Nogales, Sonora
}

\author{
Institutional trust and structural violence in Nogales, Sonora
}

\author{
Carlos Germán Palafox Moyers* \\ Joel Enrique Espejel Blanco** \\ José Ángel Valenzuela García**
}

Resumen: ${ }^{1}$ en México, la violencia se ha incrementado exponencialmente en los últimos años; representa un constructo social con elementos multifactoriales y multicausales que, para erradicarse se deben contener y controlar con medidas de prevención y participación ciudadana. El objetivo de este artículo es determinar la influencia de la acción colectiva y la cooperación y también la cohesión e inclusión social sobre la confianza institucional, y a su vez sobre la violencia estructural generada por el proceso de inmigración y deportación en Nogales, Sonora. Para contrastar las hipótesis formuladas se aplicó un instrumento estadístico a una

* Doctor en ciencias económicas y profesor-investigador del Departamento de Economía, Universidad de Sonora. Blvd. Luis Encinas y Rosales, colonia Centro, Hermosillo, Sonora, México, C. P. 83000. Teléfono: (662) 259 2167. Correo electrónico: economia@guaymas. uson.mx

** Doctor en ciencias económicas y empresariales y profesor-investigador del Departamento de Economía, Universidad de Sonora. Blvd. Luis Encinas y Rosales, colonia Centro, Hermosillo, Sonora, México, C. P. 83000. Teléfono: (662) 259 2167. Correo electrónico: jespejel@pitic. uson.mx

*** Doctor en ciencias económicas y profesor-investigador del Departamento de Economía, Universidad de Sonora. Blvd. Luis Encinas y Rosales, colonia Centro, Hermosillo, Sonora, México, C. P. 83000. Teléfono: (662) 259 2167. Correo electrónico: jvzla@pitic.uson.mx

1 Los autores agradecen el apoyo financiero de la Secretaría de Gobernación, la Comisión Nacional para Prevenir y Erradicar la Violencia contra las Mujeres y el Subsidio para la Seguridad en los Municipios. 
muestra aleatoria-estratificada en las colonias más violentas de esta ciudad fronteriza. Los resultados demostraron que la confianza y solidaridad de los habitantes es mayor hacia las instituciones públicas: gobierno local, central e instancias policiacas; en cambio, no confían tanto en las instituciones educativas (profesores) y de seguridad social (enfermeras y médicos). Sin embargo, a partir de un análisis de medias aritméticas, con muestras separadas por rango de edad, se demostró que en todas las categorías de edad se confiaba más en las enfermeras y médicos y los profesores. También, se puso de manifiesto que tanto hombres como mujeres confían más en los trabajadores de la salud y la educación.

Palabras clave: capital social; confianza institucional; violencia estructural; participación ciudadana; modelo de mínimos cuadrados parciales (PLS).

Abstract: in Mexico, violence has increased exponentially in recent years. It is a social construct with multifactorial and multicausal elements that must be contained and controlled through prevention measures and citizen participation in order to eradicate it. The objective of this article is to determine the influence of collective action and cooperation, as well as social cohesion and inclusion on institutional trust and, in turn, on structural violence generated by the process of immigration and deportation on the northern border, specifically in the city of Nogales, Sonora. To test the hypotheses, a statistical instrument was applied to a randomstratified sample in the most violent neighborhoods of this border city. Results demonstrated that people show greater trust and solidarity towards public institutions, mainly to local and central governments and law enforcement agencies; however, they show less trust in educational (teachers) and social security institutions (nurses and doctors).

Key words: social capital; institutional confidence; structural violence; citizen participation; partial least squares (PLS) models. 


\section{Introducción}

En México, la violencia se incrementó sustancialmente a partir de 2007, sobre todo la del crimen organizado, en diversos estados y más en la frontera norte (Guerrero-Gutiérrez 2010). En las últimas décadas el aumento de la población fronteriza es considerable, lo que ha generado una dinámica nueva, que incide en la vida cotidiana de los habitantes de la región, en especial en los ingresos y empleos, el trasporte, el medio ambiente, la inseguridad y la violencia. La apertura comercial y de inversión, a través del Tratado de Libre Comercio entre México, Estados Unidos y Canadá es uno de los factores que ha llevado a esta situación, y la cercanía de México con el mercado más importante y activo del mundo ha modificado los aspectos culturales, sociales y económicos de la región. La dinámica de los procesos de integración impacta a la economía formal y a la de las organizaciones delictivas, y con ello aumenta la violencia.

Según González-Corona (2009), la aglomeración en algunas regiones ha desplazado las rutas tradicionales del crimen organizado ante el debilitamiento del capital social, lo que ha provocado que se trasladen hacia otros espacios con menor población que le proveen itinerarios nuevos, que se afianzan con rapidez, como es el desierto de Altar, en Sonora (Anguiano-Téllez y Trejo-Peña 2007).

Estos "nuevos procesos" han generado muchos estudios sobre temas económicos y sociales, demográficos y culturales de la frontera norte, la mayoría relacionados con el proceso de integración entre los dos países. Sin embargo, son pocos los que abordan el fenómeno del crimen organizado y también los que analizan la violencia estructural en esta zona. Por ello, es importante resaltar que ésta sólo es una parte de un concepto más amplio. Desde una perspectiva socioeconómica, la violencia es un constructo del ser humano en sociedad con elementos multifactoriales y multicausales, que surgen por comisión u omisión de diversos actores sociales, económicos e incluso políticos y que son susceptibles de contenerse, reducirse o erradicarse. La violencia no es un mal que vino del más allá, sino una creación humana y, como tal, puede contenerse con medidas de prevención social (González-Delgado 2012; Palafox-Moyers et al. 2013).

El primer Informe regional de desarrollo humano sobre seguridad ciudadana, elaborado por el Programa de las Naciones Unidas para 
el Desarrollo (PNUD 2013), destaca que los latinoamericanos tienen miedo, y el temor principal no es, como podría creerse, a los avances del narcotráfico o las drogas, sino a los delitos comunes como los asaltos en las calles, el robo de casas, violaciones, feminicidios, pandillerismo e infanticidios, así como la intimidación ejercida por los policías e instituciones de gobierno, la llamada violencia institucional. Si bien el narcotráfico o la drogadicción son algunos de los principales problemas de la sociedad mexicana, más allá de las dificultades que enfrenta la región, hay un conjunto de amenazas que merecen igual o mayor atención.

Durante su administración, Felipe Calderón Hinojosa (20062012) colocó la lucha contra los grupos criminales en el centro de la agenda y, a pesar de ello, de acuerdo con las cifras del Sistema Nacional de Seguridad Pública (2013), el promedio mensual de los delitos federales se incrementó en 74 por ciento; además, en 24 entidades aumentó el de víctimas de secuestro. Ante la estrategia fallida del gobierno calderonista para detener la violencia en el país, es necesario atender las razones estructurales de la inseguridad, y analizar las variables de riesgo que se convierten a su vez en factores de propensión a la violencia y la criminalidad en un territorio específico.

De acuerdo con Herrera-Laso (2012), las variables socioeconómicas de mayor relevancia son la pobreza, la marginación y la infraestructura física precaria; y entre las que inciden en la criminalidad y la violencia están las pandillas, la delincuencia, las armas, las drogas y el consumo de alcohol. Además, se vinculan con el ambiente institucional y de organización social, como la escasa presencia de la autoridad, la poca organización y cohesión social, la ausencia de la cultura de la legalidad y la impunidad.

Abordar el concepto de violencia en la zona fronteriza se vuelve más complejo, porque los ciudadanos y las instituciones pertenecen a dos países, y cualquier acción no coordinada podría generar una respuesta explosiva, sobre todo en México. El caso más representativo es la política antiinmigrante de Arizona, reflejada en la Ley SB1070, la cual criminaliza la inmigración indocumentada y no sólo viola los derechos humanos sino que lleva implícita una violencia institucional contra los indocumentados y su entorno regional; ha logrado la expulsión de personas hacia México (Servicio de Inmigración y Control de Aduanas 2013). 
Según el Instituto Nacional de Migración (INM 2013), en 2008 fueron deportados 306 mil indocumentados, y 329 mil en 2010, de los cuales más de la mitad (195 mil) fueron catalogados como criminales. En 2012 se expulsó a 369 mil personas, en 2013 a 332 614, en 2014 a 242905 y en 2015 a 227 038. Además, la política de seguridad nacional de Estados Unidos hacia su país vecino sigue siendo fortalecer los muros, con una serie de implementos tecnológicos que conforman una barrera virtual de la zona, con el objeto de establecer mayores medidas de control y contención, con el reforzamiento de la vigilancia fronteriza debido al tráfico de drogas y a la migración (González-Reyes 2009). De acuerdo con datos de la Casa Blanca, Estados Unidos gasta 18 mil millones de dólares al año para proteger del cruce migratorio su frontera sur, cuyo cierre es un factor detonante para la violencia en la franja fronteriza mexicana (Pérez Silva 2013).

En México, la política social, principalmente la diseñada para la frontera norte, es insuficiente para garantizar los derechos sociales básicos como la educación, la salud, y la vivienda de un sector muy importante de la población, lo que conforma grandes áreas de pobreza, marginación y exclusión social. Los programas para la atención de niños y jóvenes son insuficientes, las oportunidades para mejorar su condición social son escasas o nulas, y no hay espacios comunitarios que permitan el trabajo colectivo y la asociación como premisa para la cohesión social. Tampoco existen programas que fomenten la cultura de la legalidad y la procuración de la justicia. Todas estas variables coadyuvan al brote de la violencia.

Asimismo, el proceso de integración debió alterar la forma de organización del crimen organizado en esta región. La frontera SonoraArizona ha sido históricamente una ruta importante para el tráfico ilegal de estupefacientes y de personas; su orografía desértica y la amplitud de la zona y de las rutas que comunican con el país vecino la convirtió en un espacio ideal para las organizaciones delictivas. La disputa por el territorio comenzó en el año 2000, y alcanzó su cúspide en 2007, con un incremento sin precedentes de la violencia. Para Mendoza-Rockwell (2012), es muy probable que las grandes trasformaciones se relacionen con el incremento en la tasa de homicidios de la región, que son: a) la privatización de las rutas del tráfico de drogas a través de la frontera; b) el reclutamiento de una burocracia 
de jóvenes como asalariados de los grupos delictivos y c) el control de las organizaciones dedicadas al tráfico de drogas y al de inmigrantes indocumentados. El resultado de estas trasformaciones es lo que la autora denomina proceso de cartelización, que hace que las entidades locales, tanto formales como informales, pierdan el control de los recursos y sean manejados por un ente exterior.

Los factores internos y externos propician una escalada de la violencia, en especial en el desierto de Altar, Sonora. A partir de 2009, el territorio más disputado por el crimen organizado fue el municipio de Nogales, donde se registraron 219 homicidios ligados a él, cifra que representa 95 por ciento del total de asesinatos en la región del desierto de Altar (Secretaría Ejecutiva de Seguridad Pública 2013). Otro indicador de la importancia de este municipio en la ruta del trasiego ilícito, es que en 2010 la Policía ministerial descubrió dos narcotúneles, uno en Nogales, Sonora, y el otro en Nogales, Arizona, donde también ha habido enfrentamientos entre los grupos más poderosos del crimen en México. Estos factores perturban la sana convivencia de las familias residentes en la frontera, lo que suscita sentimientos de inseguridad y miedo por cualquier situación que altere el orden social, aunque sea en forma mínima. Con base en lo anterior surgen los siguientes interrogantes: ¿qué opinan los habitantes de Nogales sobre la violencia y la inseguridad? ¿Qué esperan de las instituciones, en el diseño de políticas públicas sobre la inseguridad y violencia? ¿Cómo se organiza la sociedad civil para enfrentar este fenómeno?

En este trabajo, el objetivo fue escuchar la opinión de la sociedad civil del municipio de Nogales, que vive cada día el calvario de la violencia y la inseguridad de las colonias, sobre todo donde ocurre la mayor incidencia de delitos; y conocer sus formas de organización, si es que las tiene, para hacer frente a la violencia y la inseguridad.

Con la medición de la confianza institucional en cada colonia encuestada se cuantificaron los niveles de violencia estructural. La acción colectiva y la cooperación son centrales para medir el esfuerzo de toda la comunidad, así como la cohesión e inclusión social. Asimismo, al conocer los niveles de conflicto y violencia, se identificaron los espacios de sociabilidad de algunas colonias de Nogales. Esto proporciona los elementos para diseñar políticas públicas relativas a la seguridad ciudadana, así como proponer otras binacionales que per- 
mitan disminuir la tensión generada por las fuerzas internas y externas de la región en estudio. Se puede partir de lo anterior y retomar la definición de capital social de Putnam:

[...] el capital social consiste en el activo históricamente acumulado por una sociedad a partir de la acción organizada de sus miembros (individuos o colectivos), sobre la base de determinadas normas sociales de cooperación, la interiorización de varios valores (confianza, solidaridad, reciprocidad), y la existencia de un tejido social o 'redes de compromiso', permitiendo tal capital social una mayor eficacia en la consecución del bienestar $(1993,167)$.

Con base en la contextualización y la conceptualización del capital social, se pretende conocer: a) cómo influye la acción colectiva y la cooperación en la confianza institucional; b) cómo incide la cohesión y la inclusión social en la confianza institucional y c) cómo influye la confianza institucional en la violencia estructural (económica y social), según la percepción de los ciudadanos de las colonias más violentas de Nogales.

El primer apartado del artículo consta de una introducción sobre el tema. En el segundo se desarrollan las teorías sobre las violencias, así como los factores que llevan a detonarlas y los que deberían contenerlas; además, se muestran trabajos que abordan el fenómeno de la violencia en la frontera norte de México, reflejada en la coacción que sufren las y los trabajadores de la industria maquiladora de exportación y los jóvenes que radican en la región. En el tercero se describen los delitos y las colonias con mayor incidencia en Nogales. En el cuarto se desarrolla el diseño de la metodología y se interpretan los resultados, y al final se presentan las conclusiones y las recomendaciones sobre políticas públicas.

\section{Revisión de la literatura Violencia}

Hay consenso entre la mayoría de los analistas para definir la violencia como el uso de la fuerza física o psicológica con intención de hacer 
daño o restringir la libertad de las personas, de manera recurrente, con un fin predeterminado y como una forma de resolver los conflictos (Arriagada y Godoy 2000). Además, la violencia y los aspectos cercanos a ella, como la agresión y la frustración, obedecen a un conjunto complejo de elementos psicológicos, sociales y culturales.

De acuerdo con Massolo (2005), la violencia se caracteriza por tres componentes: a) intencionalidad del uso de la fuerza o poder; b) la generación de daño y c) el fin subyacente en el ejercicio de alguna forma de poder, tanto en ámbitos públicos como privados. El mismo autor considera la conveniencia de distinguir entre los conceptos de violencia de género y violencia doméstica, ya que el uso de la fuerza física contra las mujeres ocurre en espacios distintos, y no sólo afecta al género femenino. La violencia doméstica se restringe al ámbito privado, y oculta su carácter político, en tanto que la violencia de género tiene lugar tanto en el espacio doméstico como fuera de él. Además, está documentada la relación entre ambos fenómenos, ya que, por ejemplo, la violencia doméstica puede tener implicaciones en la que se ejerce en la calle, como es el caso de los feminicidios.

También es importante considerar a la violencia urbana, para Massolo (2005) se llama así porque ocurre dentro de los límites de las ciudades, según sean los parámetros para definirlas, y afirma que los procesos de urbanización destacan su carácter violento por la desigualdad social, la segregación espacial, la pobreza y la falta de planeación, así como por la ineptitud, la corrupción y el autoritarismo que caracteriza a los gobiernos locales y nacionales. Sin embargo, puesto que las administraciones municipales están más próximas a la ciudadanía, son las que tendrían que iniciar con programas que hagan énfasis en la cultura de la prevención, mediante el diseño e implementación de políticas públicas de corte trasversal.

Por otra parte, la violencia es un fenómeno multidimensional, que se ejerce en ámbitos públicos y privados, pero que es de varios tipos y modalidades, tiene variables relacionadas, motivos, agentes involucrados (individuos que la padecen y otros que la provocan) y relaciones entre ellos. Las violencias se clasifican de acuerdo con quien comete la acción u omisión, las características de las víctimas, la forma en que se ejerce el acto violento, su naturaleza, su origen y el lugar donde se lleva a cabo, así como con las relaciones que guardan entre sí 
(González-Delgado 2012). Con ello se busca detectar los elementos percusores que favorecen que las violencias se desarrollen, se generalicen y se profundicen. Así como los factores que contribuyen a crear resiliencia, esto es, capacidad para enfrentar contextos y situaciones adversas, el resistir o evitar el surgimiento de la violencia en la familia, el trabajo, la comunidad y la escuela. También se busca encontrar factores que contribuyan a su contención (Palafox-Moyers et al. 2013).

Para comprender mejor cómo ocurre la violencia social se requiere estudiar, además de sus tipificaciones, a las víctimas y a los victimarios, y también los factores asociados al fenómeno. Aunque existen diversos enfoques sobre el tema, por cuestiones de espacio, aquí sólo se hace referencia a un par de ellos. Los investigadores Arriagada y Godoy (2000) clasifican los factores de riesgo asociados a la violencia, en tres grupos: a) los relacionados con la posición y situación familiar y social de las personas: sexo, edad, educación, socialización en la violencia, consumo de alcohol y drogas; b) los sociales, económicos y culturales: desempleo, pobreza, hacinamiento, desigualdad social, violencia en los medios de comunicación y cultura de la violencia y c) los contextuales e institucionales: guerra, tráfico de drogas, corrupción, disponibilidad de armas de fuego, festividades y cierre de fronteras.

Por su parte, Buvinic et al. (1999) también distinguen tres aspectos asociados con la violencia: a) los individuales, patrones que inciden en la violencia doméstica y social: género, edad, características biológicas o fisiológicas, educación, clase social, situación laboral, uso de drogas o alcohol y haber sufrido o presenciado abuso físico en la infancia; b) los familiares, elementos como tamaño del hogar, grado de hacinamiento, historia de violencia familiar, dinámica y normas de convivencia (autocrática o democrática) y otros como ingreso per cápita del hogar, la pobreza y la incapacidad para cumplir el rol de padres, cuyo efecto puede ser marginal en la violencia doméstica y social y c) los comunitarios y sociales, que interactúan con los factores individuales y familiares.

Por la contextualización y las aportaciones planteadas, la problemática de la violencia y la criminalidad en Nogales debe abordarse en el marco de factores económicos, sociales, demográficos y culturales, que son clave en la historia de esta ciudad, además de los componentes actuales como la crisis global y la nacional, que han incidido en los procesos de pobreza, desigualdad y exclusión social. En este 
caso, el análisis se centra en la violencia estructural, definida como una situación en la que se produce un daño en los satisfactores de las necesidades básicas de la población, como falta de alimentos, salud, educación y libertad.

Estudios sobre la violencia en la frontera norte de México

Entre las investigaciones principales sobre la violencia en la frontera norte de México destaca el trabajo coordinado por Cervera-Gómez (2005), en el cual se analiza, por medio de un diagnóstico geo-socioeconómico, el estado actual del contexto urbano y social de Ciudad Juárez, Chihuahua; a través de varios estudios sobre temas sociales y urbanos como demografía, migración, educación, salud, desarrollo urbano, violencia, mercados de trabajo y economía y bienestar.

A su vez, la obra coordinada por Monárrez-Fragoso y TabuencaCórdoba (2007) ofrece puntos clave para entender varias cuestiones que al final desembocan en una misma idea: la violencia de género. Aborda el feminicidio, la violencia doméstica, el feminismo y las formas de participación política de las mujeres en relación con los temas anteriores. El estudio plantea cómo cada uno de estos fenómenos sociales restringe, dificulta y frena el avance y el desarrollo de las mujeres en la franja fronteriza norte de México.

Asimismo, Monárrez-Fragoso y García de la Rosa (2008) resaltan que en los últimos años la violencia y la inseguridad pública afectan la convivencia y el desarrollo sano y armónico de la frontera norte mexicana, aunado a la falta de un Estado de derecho y de una justicia expedita, que ha favorecido una carga de tribulación por la pérdida de vidas humanas y un alto costo económico, resultado del ambiente conflictivo y delictivo que se manifiesta en la misma frontera. El trabajo se sustenta estadísticamente con bases de datos y cifras sobre la inseguridad pública de las siguientes variables relevantes, que muestran la situación actual de los principales actos delictivos en las ciudades y zonas fronterizas: las víctimas, los homicidios, los robos, la denuncia de los delitos, la percepción y la confianza.

En la misma línea argumental, los autores mencionan algunos puntos clave y alarmantes, como es el número de víctimas por habitantes (Sonora y Chihuahua destacan como los estados con mayor denuncia 
de víctimas), así como las tasas de homicidios por habitantes (las de Chihuahua y Baja California son mayores al promedio nacional, incluso al fronterizo). Baja California, Sonora y Coahuila, tienen la tasa de robos más alta que la media nacional, y Tamaulipas, Chihuahua y Nuevo León tienen la más baja. En cuanto a las entidades con más delitos con condena, mayor porcentaje de personas que fueron víctimas de alguna agresión o robo y que denunciaron (o no) algún ilícito, por razones como "pérdida de tiempo", desconfianza en las autoridades y otras razones no detalladas, datos que demuestran que los y las mexicanas mayores de edad sienten miedo e inseguridad, está Baja California, que encabeza la lista, sigue Chihuahua, Tamaulipas, Nuevo León, Sonora y Coahuila. Por último, se entrelaza la confianza de la ciudadanía en relación con las autoridades locales y estatales, y para todos los casos el resultado fue "poca confianza". Como conclusión, se plantea la creación de un sistema fronterizo de seguridad pública que busque y fortalezca la certeza jurídica e impartición de justicia, cuerpos policiacos, información e inteligencia, cultura de la legalidad y prevención del delito (Monárrez-Fragoso y García de la Rosa 2008; Montero 2012).

Vega-Briones y Román-Robayo (2010) exponen el caso del ambiente laboral "sexuado", en la industria maquiladora de Ciudad Juárez, en Chihuahua, y su relación con los casi 400 feminicidios; afirman que las mujeres siguen siendo consideradas como "objetos sexuales". Reportan las maneras de violencia que los hombres ejercen contra las mujeres, a pesar del aporte económico de éstas para sus familias; la ideología de los trabajadores acerca de la contratación casi "sexual" de mujeres en las maquiladoras y cómo poco a poco se ha ido modificando la conducta y formas de pensar, para ambos géneros, ante los cambios en el contexto actual. Además, en el estudio se aborda el proceso migratorio de las mujeres de Ciudad Juárez hacia Estados Unidos, y cómo lo ven los esposos y los hombres, en general, así como el nuevo papel que asume la mujer en un mundo laboral que va más allá de la educación tradicional.

De la O y Flores-Ávila analizaron las condiciones de violencia en las que viven los jóvenes de la frontera norte. Para ello, realizaron entrevistas a trabajadores de las maquilas en Matamoros, Tamaulipas, durante 2010. Las narrativas de estos jóvenes revelan, según los autores, "una subsistencia inmersa en una espiral de violencia". En este 
contexto, remarcan, "sus cuerpos son el receptáculo que se consume, controla y vulnera, son recursos para la familia, mercancía para el trabajo de la maquila, objeto de sustracción y manipulación mediante el secuestro, la tortura, el levantón o el reclutamiento forzado por las organizaciones criminales" $(2012,11)$. Asocian la violencia con una precaria impartición de justicia, ausencia de políticas públicas de seguridad y con las formas socioculturales que se han construido en torno a este fenómeno.

\section{Situación actual en el municipio de Nogales, Sonora}

Nogales se ubica en el noroeste de Sonora; tiene una superficie de 1 $675 \mathrm{~km}^{2}$, y colinda hacia el norte con la ciudad de Nogales, Arizona, en Estados Unidos. Según el Instituto Nacional de Estadística y Geografía (INEGI 2010), en 2010, el municipio de Nogales tenía 220292 habitantes, ocupaba la tercera posición, después del de Hermosillo y Cajeme. En Nogales existe un crecimiento notable en la población debido a la industria maquiladora y a los grandes asentamientos de inmigrantes de regiones cercanas y de estados del sur de la república mexicana. En esta ciudad, las maquiladoras representan más de 55 por ciento del producto local bruto, seguido por la ganadería y el comercio.

\section{Figura 1}

Nogales, Sonora: incidencia mensual de delitos

\begin{tabular}{|l|c|}
\hline Delito & Incidencia mensual \\
\hline Robo a casa & 11 \\
\hline Robo a persona & 29 \\
\hline Robo a negocio & 2 \\
\hline Robo de vehículos & 31 \\
\hline Violación & 2 \\
\hline Violencia intrafamiliar & 25 \\
\hline Lesiones dolosas & 12 \\
\hline Homicidio intencional & 3 \\
\hline
\end{tabular}

Fuente: elaboración propia, con datos de la Secretaría Ejecutiva de Seguridad Pública (2013). 
En la actualidad, Nogales presenta un escenario de violencia que compromete la integridad y los derechos de los ciudadanos, debido a la atmósfera de inseguridad y a la alta tasa de incidencia de delitos, principalmente el robo de vehículos, a personas y la violencia intrafamiliar (véase figura 1). Las colonias identificadas como zonas peligrosas y focos rojos, donde se debe prestar atención y tomar medidas de seguridad inmediatas se incluyen en la figura 2.

\section{Figura 2}

Nogales, Sonora: colonias que representan un foco rojo según el delito

\begin{tabular}{|c|c|}
\hline Delitos & Colonias \\
\hline Robo a casa & $\begin{array}{l}\text { - Canoas; Cinco de Mayo; Fovissste; El Rodeo; } \\
\text { Esperanza; Fundo Legal; Granja; Kalitea Jardines; } \\
\text { Nuevo Nogales }\end{array}$ \\
\hline Robo a persona & $\begin{array}{l}\text { - Cinco de Mayo; Centro; Del Rosario; El Greco; } \\
\text { Esperanza; Héroes; Infonavit; Moderna; Obrera }\end{array}$ \\
\hline Robo a negocio & - Granja; Los Álamos \\
\hline Robo de vehículos & $\begin{array}{l}\text { - Bellavista; Bolívar; Centro; El Rodeo; } \\
\text { fraccionamiento California; Granja; Lomas de } \\
\text { Nogales; Lomas de Fátima; Lomas de Nogales } 2\end{array}$ \\
\hline Violación & - Los Álamos; San Miguel \\
\hline Violencia intrafamiliar & $\begin{array}{l}\text { - Bellotas; Buenos Aires; Canoas; Colinas del Yaqui; } \\
\text { Colosio; Empalme-Nogales; Héroes; Lomas de } \\
\text { Nogales; San Miguel }\end{array}$ \\
\hline Lesiones dolosas & $\begin{array}{l}\text { - Colinas del Yaqui; Flores Magón; Héroes; } \\
\text { Kennedy; Lomas de Nogales; Moderna; Nuevo } \\
\text { Nogales; Pueblo Nuevo; San Miguel; Solidaridad }\end{array}$ \\
\hline Homicidio intencional & - Granja; Lomas de Nogales; San Miguel \\
\hline
\end{tabular}

Fuente: Secretaría Ejecutiva de Seguridad Pública (2013).

Asimismo, se agrupan las colonias que representan las zonas peligrosas de la ciudad, debido al alto porcentaje de repeticiones de la incidencia de delitos (véase figura 3). 


\section{Figura 3}

Nogales, Sonora: colonias más peligrosas, según la repetición de incidencia de delitos

\begin{tabular}{|l|c|}
\hline \multicolumn{1}{|c|}{ Colonia } & Número de repeticiones \\
\hline Granja & 4 \\
\hline Lomas de Nogales & 4 \\
\hline San Miguel & 4 \\
\hline Héroes & 3 \\
\hline Canoas & 2 \\
\hline Centro & 2 \\
\hline Colinas del Yaqui & 2 \\
\hline El Rodeo & 2 \\
\hline Esperanza & 2 \\
\hline Los Álamos & 2 \\
\hline Moderna & 2 \\
\hline Nuevo Nogales & 2 \\
\hline
\end{tabular}

Fuente: Secretaría Ejecutiva de Seguridad Pública (2013).

Tras contextualizar la problemática de Nogales, es pertinente plantear un modelo causal de análisis, que relacione las principales variables del capital social: la acción colectiva y cooperación proactiva de las familias, el grado de cohesión e inclusión social, los niveles de confianza hacia las instituciones públicas, y cómo éstas repercuten en las dimensiones de la violencia estructural, para responder a los objetivos planteados en el presente trabajo.

\section{Planteamiento del modelo conceptual: formulación de hipótesis}

El Banco Mundial (2011) propone las dimensiones siguientes para determinar el capital social:

- La acción colectiva y la cooperación determinan el alcance de la participación grupal, cuando no está impuesta por una fuerza externa, es decir, es la iniciativa de los ciudadanos para tomar accio- 
nes en pro de la comunidad, lo que lleva a otro componente del capital social, la cohesión e inclusión social.

- La cohesión e inclusión social evalúa la percepción de la unidad social y la convivencia en la comunidad, en cuanto a la toma de decisiones y el acceso a los servicios públicos.

- La confianza institucional se refiere al nivel de confianza del ciudadano hacia las instituciones de gobierno, incluida la equidad de las normas, los procedimientos oficiales, la solución de controversias y la asignación de recursos.

Al tomar en cuenta las dimensiones expuestas, la confianza permitirá el desempeño de las instituciones gubernamentales, ya que es determinante para propiciar, facilitar y dar soporte a la cooperación (Millán 2006). En sí, "la confianza, las normas y redes pueden mejorar la eficiencia de la sociedad mediante la facilitación de las acciones coordinadas" (Putnam et al. 1994, 212). En este sentido, Millán (2006) demostró que hay una relación consistente entre la cooperación pública y la confianza, por medio de la solvencia cívica y arreglos institucionales y democráticos. Por tanto, es razonable pensar que el capital social, la acción colectiva y la cooperación, manifestados por la sociedad, tienen un vínculo positivo con la confianza hacia las instituciones de gobierno.

De acuerdo con la Comisión para América Latina y el Caribe (CEPAL 2007, 15), la cohesión social se define según dos preceptos relevantes:

- La eficacia de los mecanismos instituidos de inclusión social [como] el empleo, los sistemas educacionales, la titularidad de derechos y las políticas de fomento de la equidad, el bienestar y la protección social.

- Los comportamientos y valoraciones de los sujetos que forman parte de la sociedad [como] la confianza en las instituciones, el capital social, el sentido de pertenencia y solidaridad, la aceptación de normas de convivencia y la disposición a participar en espacios de deliberación y en proyectos colectivos.

En este sentido, la cohesión e inclusión social, como parte fundamental de la construcción del capital social, desde la perspectiva de 
los mecanismos, los comportamientos y las valoraciones de la sociedad, tiene un efecto positivo sobre la confianza en las instituciones gubernamentales, debido a la capacidad de sus ciudadanos para coordinar acciones y resolver problemas de seguridad social (salud), hasta de desarrollo económico (Millán 2006). De León-Beltrán y Velásquez $(2012,49)$ argumentan "que la confianza y la cohesión se mueven en la misma dirección: es muy difícil concebir una sociedad en la que haya cohesión sin confianza. Un aumento en la cohesión social, viene acompañado de un aumento de la confianza”.

Según las argumentaciones teóricas planteadas, es razonable formular las siguientes hipótesis de investigación:

1. Existe una influencia positiva y significativa de la acción colectiva y cooperación (capital social) sobre la confianza institucional que perciben los habitantes de las colonias más violentas de la ciudad de Nogales, Sonora.

2. Existe una influencia positiva y significativa de la cohesión e inclusión social (capital social) sobre la confianza institucional que perciben los habitantes de las colonias más violentas de la ciudad de Nogales, Sonora.

La violencia estructural se refiere a la situación en la que se produce un daño en la satisfacción de las necesidades básicas de la población, como la falta de alimentos, de salud, de educación y de libertad; resultado de la estratificación social diseñada por la administración pública, así como la globalización indiscriminada, basada en el libre mercado (Huerta-González 2009).

Con la violencia estructural presente, las instituciones y los sistemas crean disparidades entre los individuos y los grupos, y con ello eliminan toda oportunidad de movilidad social, esto es, que las familias vivan con dignidad y en mejores condiciones. Si bien este tipo de violencia no es directa, puede provocar una indirecta, como respuesta a la exclusión de los sistemas sociales, políticos y económicos de más de la mitad de la población mexicana (Consejo Nacional de Evaluación de la Política de Desarrollo Social, Coneval 2012). En consecuencia, la ciudadanía tiene "poca confianza" en las autoridades locales y estatales (Monárrez y García de la Rosa 2008). En la 
misma línea argumental, González de la Vega et al. (2010) demostraron estadísticamente que la confianza interpersonal genera efectos positivos en la confianza hacia las instituciones públicas y políticas, mientras que la situación económica-familiar y los valores materialistas y posmaterialistas, producen uno negativo hacia ella. Es decir, cuando los ciudadanos perciben mayor violencia estructural, ante la carencia de ingresos económicos, se incrementará su desconfianza en las instituciones gubernamentales, por la falta de respuesta oportuna y pertinente para resolver los problemas públicos. Al respecto, Salama (2008) muestra que las sociedades latinoamericanas tienen una gran desconfianza hacia las instituciones, debido a la exclusión social en la que viven, aunada a la insuficiencia de las políticas públicas para enfrentar las profundas desigualdades; estos dos factores son determinantes en el desarrollo de la violencia.

\section{Figura 4}

Modelo conceptual: relaciones causales entre los constructos analizados

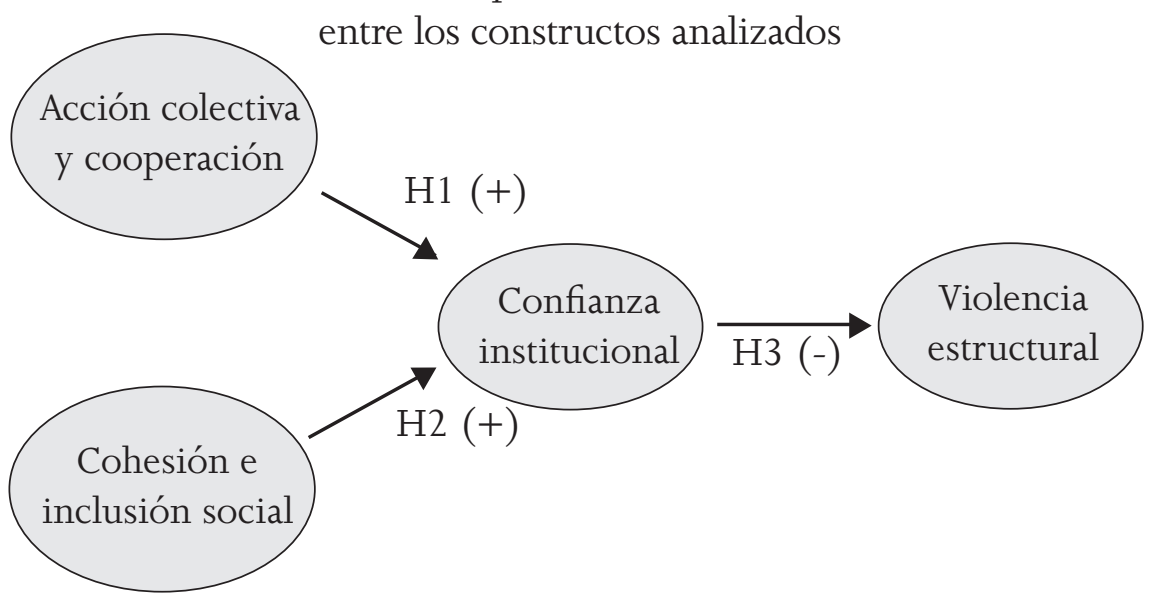

Nota: para calcular la relación causal-confianza-institucional y violencia estructural, se invirtió el sentido de la escala de medida de confianza institucional, por ende, a mayor desconfianza en las instituciones, se percibe más violencia estructural.

Fuente: elaboración propia.

En este sentido, se requiere incrementar la confianza hacia las instituciones gubernamentales, por medio de la cooperación colectiva 
y la inclusión social en la toma de decisiones de la ciudadanía, en cuanto a los servicios públicos básicos y los programas sociales, lo cual aumentaría su calidad de vida por medio de la movilidad social. Por tanto, entre mayor sea la confianza institucional, mediada por la acción colectiva-cooperación y la cohesión e inclusión social, menor será la violencia estructural que perciben los ciudadanos que habitan en las colonias más conflictivas en Nogales. Esto lleva a plantear la tercera hipótesis de trabajo: existe una influencia negativa y significativa de la confianza institucional (capital social) sobre la violencia estructural (social y económica) que perciben los habitantes de las colonias más violentas de la ciudad de Nogales, Sonora (véase figura 4).

\section{Diseño metodológico}

Investigación cuantitativa

El cuestionario estructurado que se utilizó fue el del Banco Mundial (2011), con escalas de Likert de 5 puntos. Se aplicó en las colonias más peligrosas, según el número de repeticiones de incidencia de delito en Nogales. La muestra se determinó con el semáforo delictivo de la ciudad de Nogales, de la Secretaría Ejecutiva de Seguridad Pública (2013).

Metodología de análisis

Para el análisis estadístico de los datos se usaron los modelos de ecuaciones estructurales con la técnica mínimos cuadrados parciales (PLS, por sus siglas en inglés), y se aplicó el paquete informático estadístico SmartPLS 2.0 (Ringle et al. 2005).

Criterios de la selección de la muestra

Fueron 103 las entrevistas realizadas a personas mayores de 18 años, y que viven en manzanas pertenecientes a zonas de atención prioritaria, en las áreas geoestadísticas básicas 1829, 1265, 0958, 102A, $141 \mathrm{~A}, 166 \mathrm{~A}, 0731$ y 0835 , de Nogales. Las zonas analizadas se ubican al norponiente de la ciudad, porque es donde hay más habitantes 
con grado de marginación urbana y grado de rezago social urbano (Secretaría de Desarrollo Social, SEDESOL 2015) y problemas de inmigración y deportación (INM 2013) (véase figura 5).

\section{Figura 5}

Ficha técnica

\begin{tabular}{|l|l|}
\hline Características & Datos \\
\hline Ámbito geográfico & $\Rightarrow$ Municipio de Nogales, Sonora \\
\hline Universo & $\begin{array}{l}\Rightarrow \text { Personas mayores de } 18 \text { años que viven en las } \\
\text { zonas con mayor incidencia delictiva en Nogales, } \\
\text { con base en el semáforo delictivo de la Secretaría } \\
\text { Ejecutiva de Seguridad Pública (2013) }\end{array}$ \\
\hline Metodología & $\begin{array}{l}\Rightarrow \text { Entrevista personal mediante cuestionario } \\
\text { semiestructurado (Banco Mundial 2011) }\end{array}$ \\
\hline Procedimiento de muestreo & $\Rightarrow$ Aleatorio-estratificado \\
\hline Tamaño muestral & $\begin{array}{l}\Rightarrow 103 \text { cuestionarios válidos, 500 por remuestreo } \\
\text { (técnica bootstrap) }\end{array}$ \\
\hline Fecha del trabajo de campo & $\Rightarrow$ Octubre de 2013 \\
\hline
\end{tabular}

Fuente: elaboración propia.

\section{Análisis estadístico de los datos}

Para contrastar las hipótesis y hacer el análisis estadístico de los datos, se realizaron las actuaciones siguientes.

Validez de contenido: revisión exhaustiva de la literatura especializada en economía social y violencia, para sustentar cada indicador de medida del modelo planteado.

Validez aparente: el instrumento estadístico del Banco Mundial se sometió a un panel de expertos en la materia, para clarificar los indicadores (reactivos), y adaptarlos al contexto de análisis. Los indicadores de medida definitivos usados para los análisis estadísticos de los datos aparecen en la figura 6. 


\section{Figura 6}

\section{Indicadores de medida del modelo}

\begin{tabular}{|c|c|}
\hline Constructo & Indicadores \\
\hline \multirow{4}{*}{$\begin{array}{l}\text { Acción colectiva } \\
\text { y cooperación }\end{array}$} & $\begin{array}{l}\text { AC5.5. Organización colectiva y cooperación } \\
\text { ante la falta de suministro de agua }\end{array}$ \\
\hline & $\begin{array}{l}\text { AC.5.6. Organización comunitaria ante problemas } \\
\text { de enfermedad o muerte de alguien en la colonia }\end{array}$ \\
\hline & $\begin{array}{l}\text { AC.5.7. Personas en la colonia que contribuyen } \\
\text { a las actividades de la comunidad }\end{array}$ \\
\hline & $\begin{array}{l}\text { AC5.8. Personas en la colonia que contribuyen con tiempo } \\
\text { o con dinero para el logro de objetivos de desarrollo comunes }\end{array}$ \\
\hline \multirow{2}{*}{$\begin{array}{l}\text { Cohesión e } \\
\text { inclusión social }\end{array}$} & IS6.1. ¿Cómo clasificaría la unidad social de esta colonia? \\
\hline & $\begin{array}{l}\text { IS6.10. ¿Cuánta fuerza tiene el sentimiento } \\
\text { de unión o cercanía en su colonia? }\end{array}$ \\
\hline \multirow{5}{*}{$\begin{array}{l}\text { Confianza } \\
\text { institucional }^{*}\end{array}$} & CS4.3D. Confianza en los funcionarios del gobierno local \\
\hline & CS4.3E. Confianza en los funcionarios del gobierno central \\
\hline & CS4.3F. Confianza en la policía \\
\hline & CS4.3G. Confianza en los profesores \\
\hline & CS4.3H. Confianza en las enfermeras y médicos \\
\hline \multirow[t]{5}{*}{$\begin{array}{l}\text { Violencia } \\
\text { estructural* }\end{array}$} & $\begin{array}{l}\text { V6.24. ¿Esta colonia es generalmente tranquila } \\
\text { o está marcada por la violencia? }\end{array}$ \\
\hline & $\begin{array}{l}\text { V6.25. ¿El nivel de violencia en esta colonia ha aumentado, } \\
\text { disminuido o se ha mantenido? }\end{array}$ \\
\hline & $\begin{array}{l}\text { V6.26. ¿Se siente seguro respecto al delito } \\
\text { y la violencia cuando está solo en su hogar? }\end{array}$ \\
\hline & $\begin{array}{l}\text { V6.27. ¿Se siente seguro cuando camina } \\
\text { por la calle después de que anochece? }\end{array}$ \\
\hline & V6.33. ¿Es alto el desempleo en su colonia? \\
\hline
\end{tabular}

* Nota: el constructo "confianza institucional" se basa en indicadores sobre la confianza de los ciudadanos hacia los representantes de las instituciones públicas (gobierno local y central, policía, profesores y médico). Para el constructo "violencia estructural" se usaron indicadores de violencia social (tranquilidad, violencia, delitos y seguridad en la colonia), así como de violencia económica (desempleo en la colonia).

Fuente: elaboración propia, con base en Banco Mundial (2011). 
Validez del modelo de medida: para depurar los indicadores de los modelos PLS, se analizaron las cargas factoriales $(\lambda)$ y la comunalidad $\left(\lambda^{2}\right)$-varianza de las variables- de cada constructo objeto de estudio. En cuanto a la fiabilidad de los indicadores, para que se acepte uno como integrante de un constructo, debe poseer una carga factorial $(\lambda)$ o correlaciones simples iguales o superiores a 0.707 (Carmines y Zeller 1979). Esto indica que la varianza compartida entre el constructo y los indicadores de éste sea mayor que la varianza del error. La comunalidad $\left(\lambda^{2}\right)$ es el cuadrado de la correlación entre las variables manifiestas y su propia variable latente. Por ejemplo, el primer indicador AC5.5 tiene una carga factorial de $\lambda=0.663$, que representa una comunalidad de $\left(\lambda^{2}\right)=0.4395$, lo que indica que 43.95 por ciento de la varianza de la variable manifiesta está relacionada con el constructo "acción colectiva y cooperación" (véase figuras 7, 8, 9 y 10).

\section{Figura 7}

Fiabilidad de los indicadores: acción colectiva y cooperación

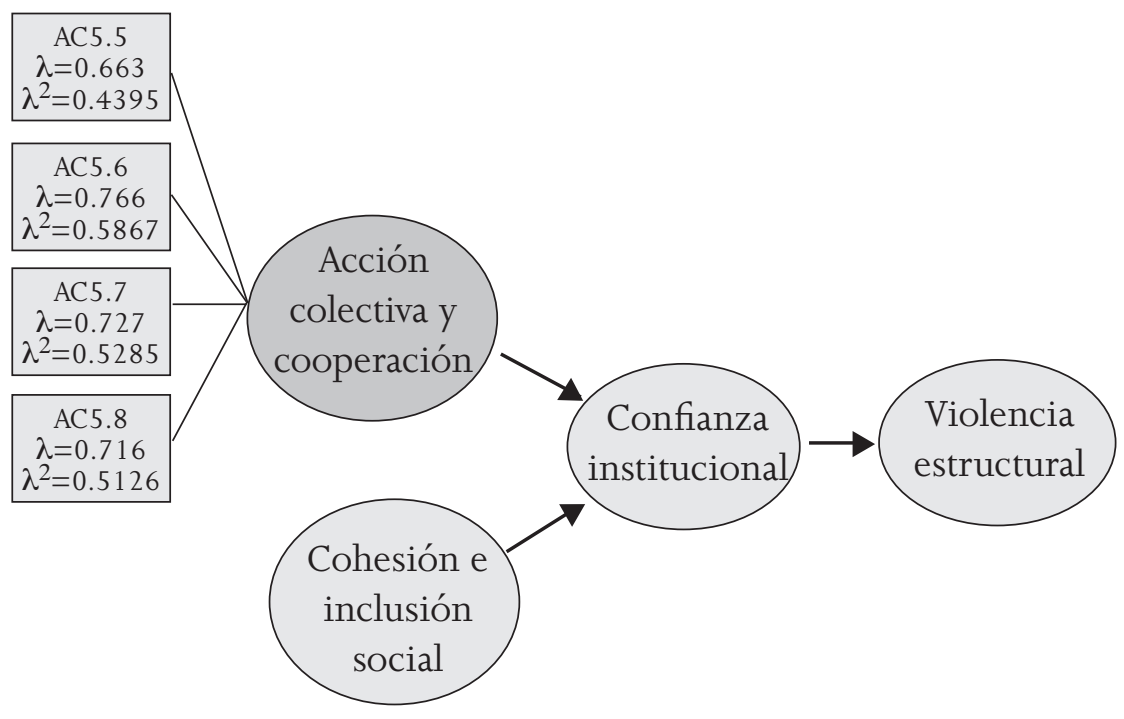

Fuente: elaboración propia. 


\section{Figura 8}

Fiabilidad de los indicadores: cohesión e inclusión social

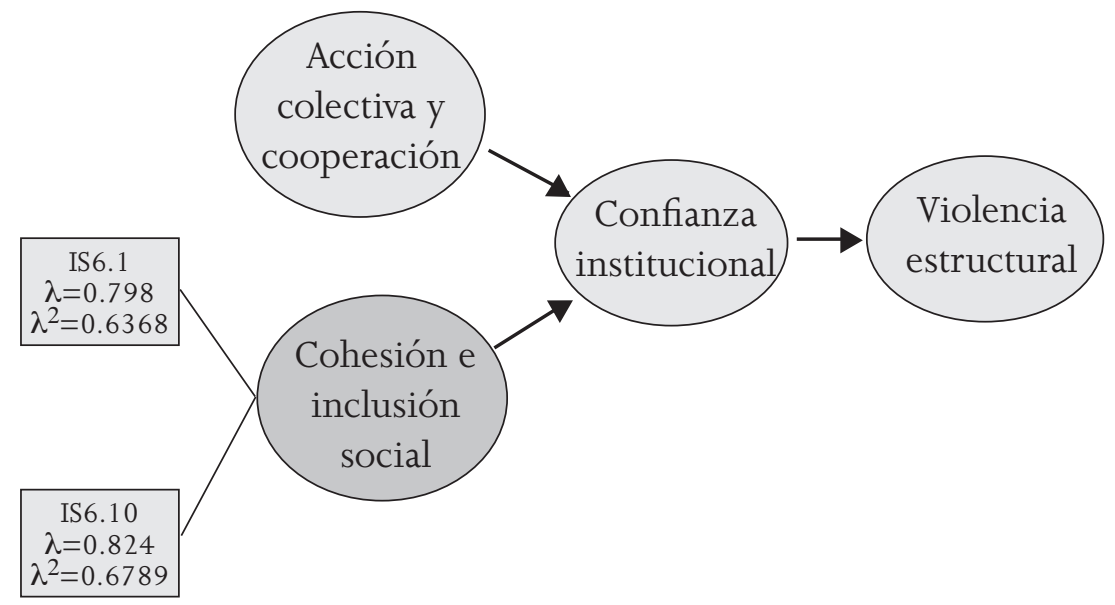

Fuente: elaboración propia.

\section{Figura 9}

Fiabilidad de los indicadores: confianza institucional

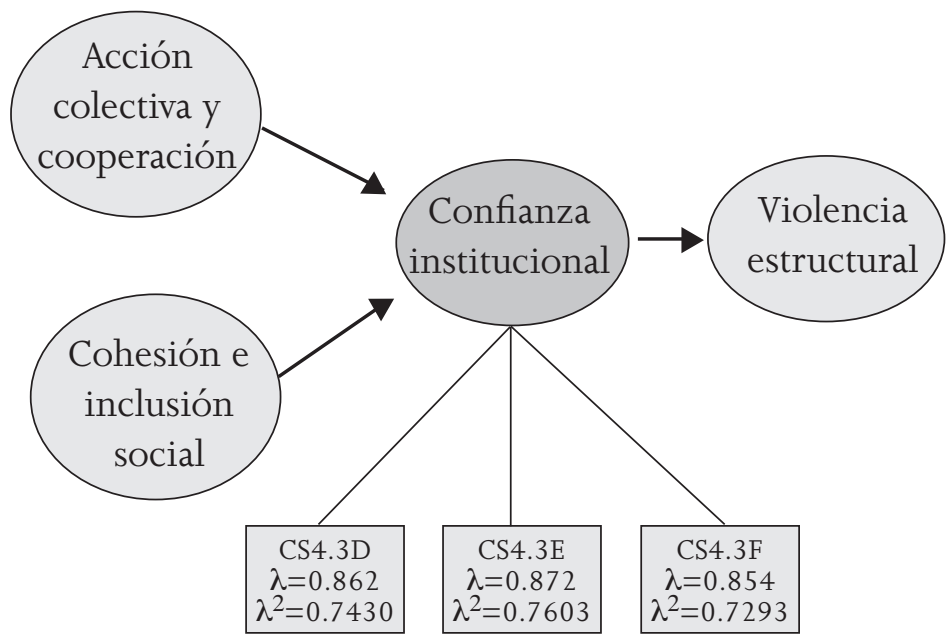

Nota: los indicadores de medida CS4.3G y CS4.3.H se depuraron, no poseen la carga factorial adecuada $(\lambda \geq 0.707)$.

Fuente: elaboración propia. 


\section{Figura 10}

Fiabilidad de los indicadores: violencia colectiva

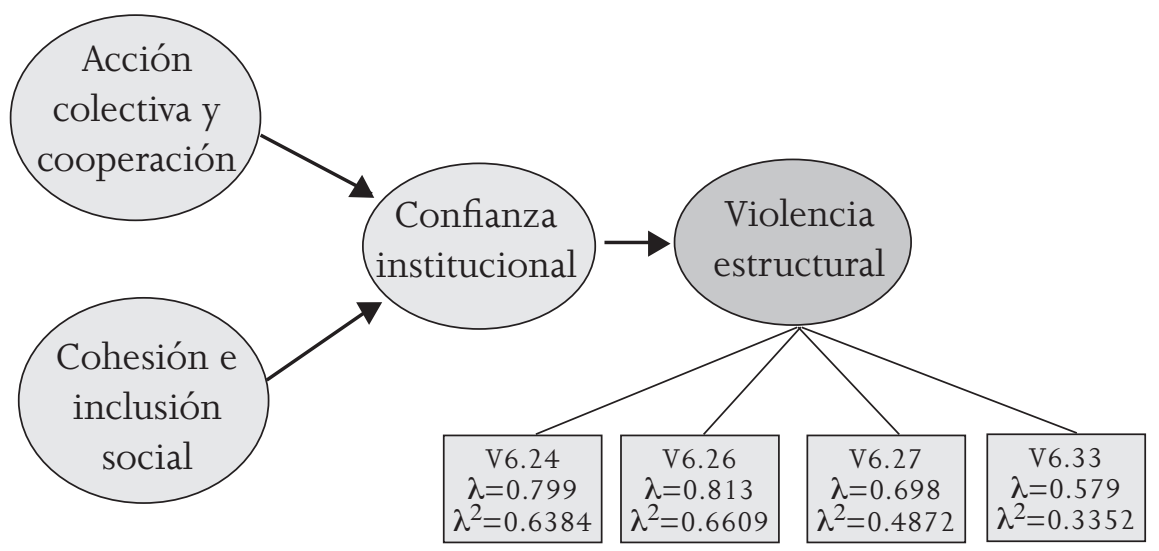

Nota: el indicador de medida V6.25 se depuró, no posee la carga factorial adecuada $(\lambda \geq 0.707)$. Fuente: elaboración propia.

Además, se cuantificaron el alfa de Cronbach $(\alpha)$, el coeficiente de fiabilidad compuesta $\left(\rho_{\mathrm{c}}\right)$ y la validez convergente, por medio del coeficiente de análisis de la varianza extraída (AVE) y la validez discriminante (véase figura 11).

\section{Figura 11}

Modelo de medida: fiabilidad del constructo

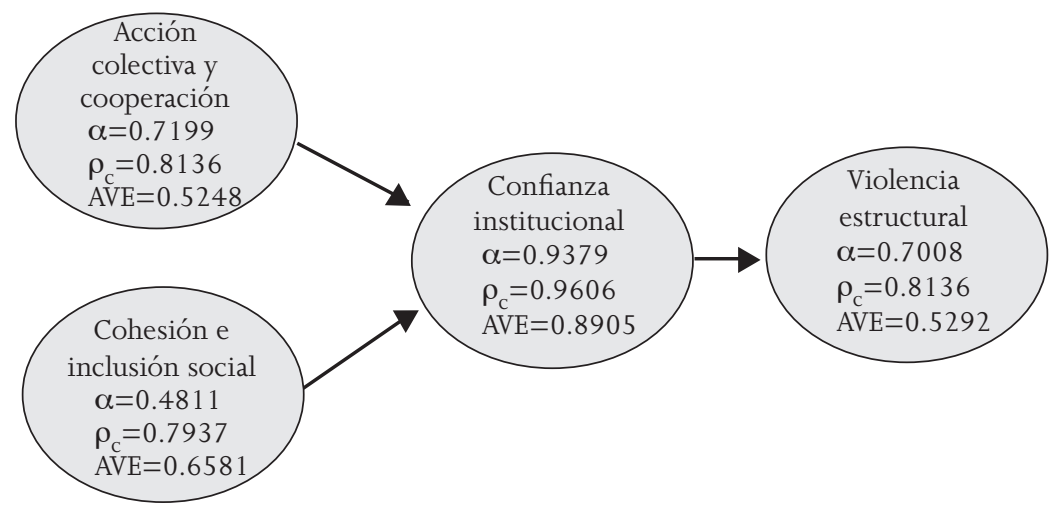

Fuente: elaboración propia. 
El alfa de Cronbach es mayor a 0.7 en todos los casos, se cumple el criterio empírico (Sanz et al. 2008). En coeficiente de fiabilidad compuesta o coeficiente rho de Spearman, todos los constructos poseen valores mayores a 0.6 (Bagozzi e Yi 1988; Chin 1998). Asimismo, todos los coeficientes de fiabilidad compuesta son superiores a los valores de alfa de Cronbach para cada uno de los constructos propuestos (Fornell y Larcker 1981); por tanto, éstos poseen una consistencia interna satisfactoria. El coeficiente AVE para todos los constructos es mayor a 0.5 (Bagozzi 1981; Fornell y Larcker 1981). Es decir, que más de 50 por ciento de la varianza se debe a sus indicadores. El análisis previo permite demostrar la validez convergente de los constructos. La validez discriminante queda comprobada si el constructo analizado está alejado significativamente de otros, con los que en teoría se encuentra relacionado (Roldán 2000).

Validez del modelo estructural: se realizaron los cálculos de la varianza explicada $\left(\mathrm{R}^{2}\right)$; los coeficientes path estandarizados $(\beta)$; la prueba StoneGeisser o valor $\mathrm{Q}^{2}$ y el índice global de bondad de ajuste (GOF, por sus siglas en inglés) (véase figura 12).

\section{Figura 12}

\section{Modelo estructural PLS}

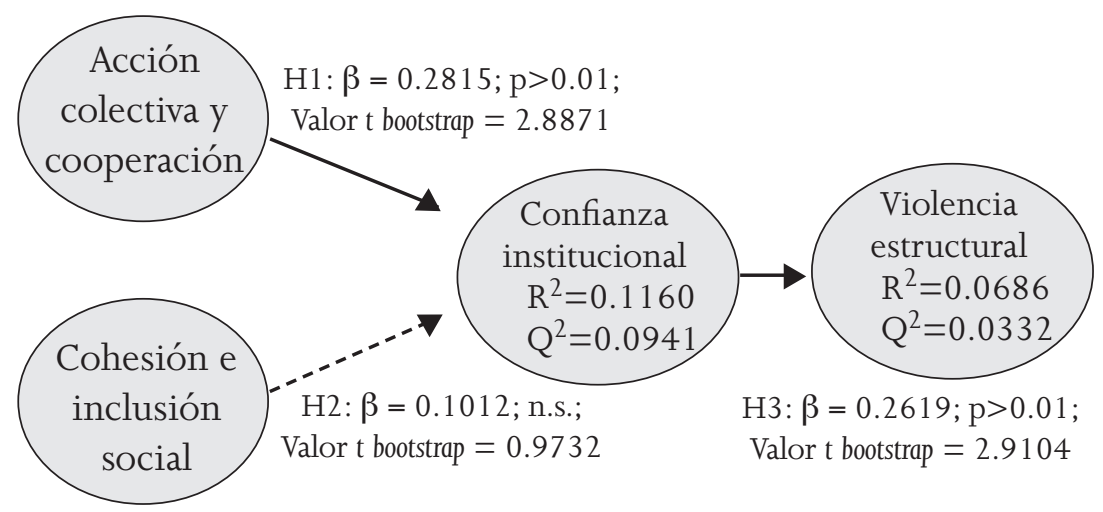

Nota: ${ }^{* * *}$ valor $\mathrm{t}>2576(\mathrm{p}<0,01),{ }^{* *}$ valor $\mathrm{t}>1960(\mathrm{p}<0,05),{ }^{*}$ valor $\mathrm{t}>1645(\mathrm{p}<0,10)$, n.s. = no significativo.

Fuente: elaboración propia. 
La varianza explicada de las variables endógenas o dependientes $\left(\mathrm{R}^{2}\right)$ debe ser igual o mayor a 0.1 (Falk y Millar 1992). A partir de este criterio empírico, todos los constructos poseen una calidad de poder de predicción aceptable. Los coeficientes path o pesos de regresión estandarizados $(\beta)$ deben alcanzar al menos un valor de 0.2 para que se consideren significativos, y situarse por encima de 0.3 (Chin 1998). Según el criterio anterior, se cumplen estadísticamente todas las relaciones causales propuestas como hipótesis, en relación con la variable latente. El parámetro $\mathrm{Q}^{2}$ debe ser mayor a cero, para que el constructo tenga validez predictiva (Chin 1998), ya que los valores por encima de cero demuestran que la predictibilidad del modelo es relevante. El $\mathrm{GOF}=\sqrt{ }(\mathrm{AVE})^{*}\left(\mathrm{R}^{2}\right)$ (Tenenhaus 2008; 2005). Por ende, al utilizar la fórmula se obtiene un GOF $=\sqrt{ }(0.6506)^{*}(0.0923)=0.2450$, dicho indicador demuestra que se tiene un buen ajuste tanto en el modelo de medida como en el estructural, y cumple con el criterio empírico de que la medida de bondad de ajuste debe variar entre 0 y 1 .

\section{Interpretación de los resultados}

La mayoría de los habitantes de Nogales opina que las colonias están marcadas por la violencia, en especial por el robo a casas habitación, a personas, robo de autos y violencia intrafamiliar, entre otros delitos. Además, señalan que las violencias se han mantenido en los últimos años, y también que el hogar sigue siendo el lugar más seguro para la mayoría. Por otra parte, expresan que se sienten inseguros al caminar por las calles, sobre todo al anochecer, ya que muchas colonias carecen de alumbrado público o no funciona.

La violencia y la inseguridad siguen siendo el calvario cotidiano para la mayoría de sus habitantes, pues el robo a casas habitación sobresale en las colonias Canoas y Cinco de Mayo; el robo a personas en la Cinco de Mayo; el robo de autos en la Bellavista y Centro y la violencia intrafamiliar en la Buenos Aires.

Por otra parte, la mayoría de las personas señalaron no pertenecer a alguna organización o colectivo, y tampoco muchos de sus familiares más cercanos y, cuando se afilian a alguno es de tipo religioso. 
También, manifestaron que hay cierta confianza entre los integrantes de la misma calle, pero no en los de la colonia o de otras.

Los habitantes de las colonias más violentas de Nogales no perciben una relación causal entre la cohesión e inclusión social y la confianza institucional, parece razonable pensar que prevalece el individualismo y el egoísmo; lo anterior pone de manifiesto que los nogalenses no mantienen la unidad social y la convivencia en la comunidad, en cuanto a la toma de decisiones y el acceso a los servicios públicos básicos que deben proporcionar las instituciones de gobierno; por tanto, se rechaza la hipótesis 2.

En relación con la variable acción colectiva y cooperación, moderada por la confianza institucional y la violencia estructural (hipótesis 1), los habitantes de Nogales confían en las instituciones públicas, principalmente en el gobierno local, central y los policías; en cambio, no perciben confianza hacia las instituciones educativas (profesores) y de seguridad social (enfermeras y médicos). Sin embargo, a partir de un análisis de medias aritméticas, con muestras separadas por rango de edad, se demostró que en todas las categorías de edad se confiaba más en las enfermeras y médicos y los profesores. También, se puso de manifiesto que tanto hombres como mujeres confían más en los trabajadores de la salud y la educación. Se encontró que en cualquier tipo de ocupación se generan mayores vínculos de confianza con las enfermeras y médicos, así como con los profesores.

Sobre la confianza institucional, los residentes de las colonias más violentas de Nogales perciben que a mayor confianza en las instituciones públicas, menor será la influencia hacia la violencia estructural, tanto social como económica, por lo anterior se acepta la hipótesis 3.

\section{Conclusiones e implicaciones gubernamentales}

Un sector importante de la sociedad de Nogales -los más desprotegidos y pobres- está flanqueado por las políticas discriminatorias de Estados Unidos, donde se criminaliza la inmigración indocumentada que, además de violar los derechos humanos, lleva implícita una violencia institucional contra los indocumentados y su entorno regional. 
El cierre de la frontera por parte de EE UU puede ser un factor detonante para la violencia en la franja fronteriza mexicana.

Por otra parte, la sociedad está sitiada por el crimen organizado y su nueva forma de conformación, denominada proceso de cartelización, hace que las entidades locales formales e informales pierdan el control de los recursos, y sean manejados por un ente externo, con lo que la violencia escala a niveles inimaginables. Además están los delitos comunes, como son los asaltos en las calles, el robo a casas habitación, las violaciones, la violencia intrafamiliar, los feminicidios, el pandillerismo y los robos patrimoniales que deterioran la convivencia y el desarrollo sano y armónico que la ciudad tenía en años anteriores.

A muchos nogalenses les aqueja la violencia estructural; la situación en la que se produce un daño en los satisfactores de sus necesidades básicas como en los alimentos, la salud, la educación y la libertad, entre otros derechos que el Estado debe garantizar, por mandato constitucional, para la población más desprotegida. Estos cuatro factores incrementan la posibilidad de tener escenarios con mucha violencia, lo que perjudica a los sectores más vulnerables de la ciudad.

La participación de la sociedad civil en esta grave situación es escasa o nula, ya que la mayoría de las personas señalan no pertenecer a alguna organización o colectivo, para diseñar políticas públicas, tal vez debido a las condiciones de pobreza-marginación-exclusión y violencia en la que se encuentra una gran parte de la población de Nogales. Por ello es importante retomar el planteamiento sobre participación ciudadana que propone Canto-Chac (2008), a favor de la que se resumen los siguientes argumentos: a) proporciona información sobre el ambiente social en el que se ejecutarán las actividades de desarrollo; b) revela de manera más eficiente las preferencias de los usuarios; c) genera aprendizajes sociales e innovación; d) fortalece a las instituciones locales; e) genera mayor credibilidad y legitimidad sobre las evaluaciones; f) contribuye a mejorar la eficiencia de las instituciones locales; g) promueve la formación de capital social; h) fortalece la competitividad sistémica de la región o localidad; i) contribuye a la formación o fortalecimiento de identidad local o regional.

Los argumentos en contra o sus contradicciones son: a) propicia la formación de elites que sesgan la participación; b) fomenta la exclu- 
sión persistente de los menos organizados para gestionar sus demandas; c) se vuelve ocasión para la "captura" de recursos e instituciones redistributivas por parte de elites locales; d) sesga las preferencias del universo de usuarios hacia las de los participantes; e) propicia la "informalización" de la política al abrir canales alternos a los de representación institucionalizada; f) limita la racionalización de las acciones gubernamentales debido a la dispersión de las demandas y g) disminuye la confianza hacia las instituciones representativas.

Al respecto, Huerta-González (2009) propone que el Estado debe retomar el manejo soberano de la política económica, con el objetivo de asegurar las condiciones productivas y financieras en pro del crecimiento sostenido con empleo pleno. Por tanto, se debe transitar urgentemente hacia un modelo de mercado con enfoque social, y a la participación del Estado en las decisiones económicas de empresarialidad social; con ello la sociedad fortalecerá la confianza en las instituciones gubernamentales, consolidará la movilidad social y empoderará a las organizaciones sociales por medio de la gobernanza y participación cívica y ciudadana, para generar acciones y proyectos beneficiosos para la comunidad.

La cohesión social, la acción colectiva, la confianza y la eficiencia institucional, como manifestaciones positivas del capital social, son elementos clave para el desarrollo económico de una región, por tanto, el desafío es trasformar el papel preponderante que juega el capital social en una comunidad, ante la indiferencia y debilidad de las instituciones gubernamentales formales (Woolcock y Narayan 1997). Al respecto, se debe reforzar el sentido de pertinencia, a partir de experiencias positivas concretas de la sociedad, la participación ciudadana activa en la toma de decisiones gubernamentales y la consolidación de los valores de la convivencia (CEPAL 2007) y, con ello implementar políticas públicas que consideren el multiculturalismo y definan ejes rectores binacionales, que se enfoquen en construir el capital social de ambos municipios fronterizos (Nogales, Sonora-Arizona), sobre todo en las zonas urbanas y rurales más violentas, desprotegidas y marginadas.

Ante la hipótesis de que la globalización estimula el círculo vicioso de pobreza-marginación-exclusión social-violencia, la sociedad mexicana en su conjunto debe participar en el diseño de la planifi- 
cación económica y social e incluir a los sectores académicos, públicos y privados, organismos gubernamentales y no gubernamentales, sindicatos, agrupaciones de productores y campesinos y centros de investigación, por citar algunos, con el objetivo fundamental y estratégico de resolver con urgencia, inteligencia y pertinencia la inseguridad que sienten los mexicanos, por medio de la recuperación de la confianza hacia las instituciones políticas, y revertir con ello la violencia estructural que tanto aqueja a México.

\section{Bibliografía}

Aguilar-Villanueva, Luis Fernando. 2009. Gobernanza y gestión pública. México: Fondo de Cultura Económica.

Anguiano-Téllez, M. E. y Alma Paola Trejo Peña. 2007. Vigilancia y control en la frontera México-Estados Unidos: efectos en las rutas del flujo migratorio internacional. Papeles de Población 3 (51): 45-75.

Arriagada, Irma y Lorena Godoy. 2000. Prevenir o reprimir: falso dilema de la seguridad ciudadana. Revista de la CEPAL 70: 107-131.

Bagozzi, Richard P. 1981. Evaluating structural equations models with unobservable variables and measurement error: a comment. Journal of Marketing Research 18 (3): 375-381.

Bagozzi, Richard P. e Youjae Yi. 1988. On the evaluation of structural equation models. Journal of the Academy of Marketing Science 16 (1): 74 94.

Banco Mundial. 2011. ¿Qué es el capital social? http://web.worldbank.org/wbsite/external/topics/extsocialdevelopment/ exttsocialcapital/0, content MDK:20187568 pagePK:210058 pi PK:210062 theSite PK:401015,00.html (9 de diciembre de 2014).

Buvinic, Mayra, Andrew Morrison y Michael Shifter. 1999. Violence in Latin America and the Caribbean: a framework for action.Technical study. Sustainable 
Development Department. Washington: Banco Interamericano de Desarrollo.

Canto-Chac, Manuel. 2008. Gobernanza y participación ciudadana en las políticas públicas frente al reto del desarrollo. Políitica y Cultura 30: 9-37.

Carmines, Edward G. y Richard Zeller. 1979. Reliability and validity assessment. Beverly Hills: Sage.

CEPAL. 2007. Cohesión social: inclusión y sentido de pertenencia en América Latina y el Caribe. CEPAL, Agencia Española de Cooperación Internacional, Secretaría General Iberoamericana, Santiago, Chile. http://segib.org/documentos/esp/cohesionsocial.pdf (8 de diciembre de 2014).

Cervera-Gómez, Luis Ernesto. 2005. Diagnóstico geo-socioeconómico de Ciudad Juárez y su sociedad. Ciudad Juárez: El Colegio de la Frontera Norte, Instituto Nacional de las Mujeres.

Chin, W. Win. 2000. Partial least square for researchers: an overview and presentation of recent advances using the PLS approach. http:/ / discnt.cba.uh.edu/chin/indx.html (25 de noviembre de 2014).

Chin, W. Win. 1998. The partial least squares approach to structural equation modelling. En Modern methods for business research, Lawrence Erlbaum Associates, editado por George Marcoulides. Nueva Jersey: Mahwah.

CONEVAL. 2012. Informe de pobreza en México 2010: el país, los estados y los municipios. México: CONEVAL.

De la O, María Eugenia y Alma Leticia Flores-Ávila. 2012. Violencia, jóvenes y vulnerabilidad en la frontera noreste de México. Revista Desacatos 38: 11-28.

De León-Beltrán, I. y Elkin Velásquez. 2012. Cohesión, confianza y seguridad: un estudio exploratorio. En Violencia y cohesión social en América 
Latina, editado por Francisco Javier Díaz y Patricio Moller. Santiago de Chile: Corporación de Estudios para América Latina.

Falk, Frank y Nancy Miller. 1992. A primer for soft modeling. Akron: The University of Akron Press.

Fornell, Claes y David Larcker. 1981. Evaluating structural equation models with unobservable variables and measurement error. Journal of Marketing Research 18 (1): 39-50.

González Corona, Rosa María. 2009. Violencia y capital social en la zona metropolitana de Tijuana. En Diagnóstico sobre la realidad social, económica y cultural de los entornos locales para el diseño de intervenciones en materia de prevención y erradicación de la violencia en la región norte, coordinado por Silvia Lopez, 139-172. México: Secretaría de Gobernación. http://cedoc.inmujeres.gob.mx/lgamvlv/conavim/tijuana.pdf\#page $=140$

González de la Vega, A., Ángel Quintanilla y Mariana Tajonar. 2010. Confianza en las instituciones políticas mexicanas: ¿capital social, valores culturales o desempeño? En La confianza en las instituciones. México en perspectiva comparada, coordinado por Alejandro Moreno, 63-97. México: Centro de Estudios Sociales y de Opinión Pública, Instituto Tecnológico Autónomo de México.

González-Delgado, Micaela. 2012. Para entender la violencia en el Estado de México: elementos para su discusión. En Memoria electrónica del IV Coloquio de investigación educativa, compilado por María de los Ángeles Trejo González y Milagros Pichardo Hernández. México: Facultad de Estudios Superiores, Acatlán de la Universidad Nacional Autónoma de México (UNAM).

González-Reyes, Pablo Jesús. 2009. Migración, criminalidad y violencia en la frontera norte de México. Revista de Criminalidad 51 (2): 47-60.

Guerrero-Gutiérrez, Eduardo. 2010. Los hoyos negros de la estrategia contra el narco. Revista Nexos 392: 27-35. http://www.nexos.com. $\mathrm{mx} / \mathrm{p}=13844$ (28 de noviembre de 2014). 
Herrera-Laso, Luis. 2012. Factores que propician la violencia y la inseguridad: apuntes para una estrategia integral de seguridad pública en México, Grupo Coppan, SC. www.seguridadcondemocracia. org (4 de noviembre de 2014).

Huerta-González, Arturo. 2009. Hacia el colapso de la economía mexicana. Diagnóstico, pronóstico y alternativas. México: UNAM.

INEGI. Censo de población y vivienda. 2010. INEGI. http://www.censo2010.org.mx/ (5 de diciembre de 2014).

INM. 2013. Síntesis 2013. Estadística migratoria. Secretaría de Gobernación, Unidad de Policía Migratoria, INM. El resumen ejecutivo del diagnóstico del Instituto Nacional de Migración. http://www.politicamigratoria.gob.mx/es_mx/sEGOB/Documentacion_y_condicion_estancia_2013 (10 de septiembre de 2014).

Monárrez-Fragoso, Julia Estela y Jaime García de la Rosa. 2008. Violencia e inseguridad en la frontera norte de México. Nóesis. Revista de Ciencias Sociales y Humanidades 17 (34): 42-65.

Monárrez-Fragoso, Julia Estela y María Socorro Tabuenca-Córdoba (coordinadoras). 2007. Bordeando la violencia contra las mujeres en la frontera norte de México. México: El Colegio de la Frontera Norte, Miguel Ángel Porrúa.

Massolo, Alejandra. 2005. Género y seguridad ciudadana: el papel y el reto de los gobiernos locales: seminario permanente sobre violencia. http//www://violenciael salvador.org.sv/documentos/junio2005/ponenciamassolo (7 de octubre de 2014).

Mendoza-Rockwell, Natalia. 2012. Crónica de la cartelización. Revista Nexos 414: 74-79. http://www.nexos.com.mx/?p=14846 (10 de septiembre de 2014).

Millán, Rene. 2006. Confianza y participación en México: ¿dimensiones de la cooperación social y de la valoración del gobierno? Revista Opinião Pública 12 (2): 211-240. 
Montero, Juan Carlos. 2012. La estrategia contra el crimen organizado en México: análisis del diseño de la política pública. Revista Perfiles Latinoamericanos 20 (39): 7-30.

Palafox-Moyers, Carlos Germán, Joel Enrique Espejel-Blanco, Benjamín Burgos-Flores y Javier Antonio García-Pequeño. 2013. Economía y violencia en el municipio de Navojoa. International Review of Business Research Papers 9 (2): 43-66.

Pérez Silva, Ciro. 2013. Muros, alta tecnología y crimen no detienen a los indocumentados. La Jornada. 8 de julio. http://www.jornada. unam.mx/2013/07/08/politica/007n1pol

PNUD. 2013. Informe regional de desarrollo humano para América Latina 20132014. Seguridad ciudadana con rostro humano: PNUD. Santiago de Chile: Centro Regional de Servicios para América Latina y el Caribe, Dirección Regional para América Latina y el Caribe.

Putnam, Robert. 1993. The prosperous community: social capital and public life. American Prospect 4 (13): 35-42.

Putnam, Robert, Robert Leonardi y Raffaella Nanetti. 1994. Para que la democracia funcione. Las tradiciones cívicas en la Italia moderna. Caracas: Editorial Galac.

Ringle, Christian M., Sven Wende y Will Alexander. 2005. SmartPLS 2.0 (M3). Hamburgo, Alemania. http://www.smartpls.de (4 de agosto de 2014).

Roldán, José L. 2000. Sistemas de información ejecutivos EIS. Génesis, implantación; repercusiones organizativas. Tesis doctoral. Universidad de Sevilla.

Salama, Pierre. 2008. Informe sobre la violencia en América Latina. Revista de Economía Institucional 10 (18): 81-102.

Sanz Blas, Silvia, Carla Ruiz Mafé y Joaquín Aldás Manzano. 2008. La influencia de la dependencia del medio en el comercio electrónico 
B2C. Propuesta de un modelo integrador aplicado a la intención de compra futura en internet. Cuadernos de Economía y Dirección de la Empresa 36: 45-76.

Secretaría Ejecutiva de Seguridad Pública. 2013. Semáforo delictivo de la ciudad de Nogales, correspondiente al mes de octubre, Secretaría de Seguridad Pública del Estado de Sonora.

SEDESOL. 2015. Programa para el Desarrollo de Zonas Prioritarias (PDZP), SEDESOL. http://www.sedesol.gob.mx/es/SEDESOL/Zonas_de_Atencion_Prioritaria_2015 (10 diciembre de 2015).

Servicio de Inmigración y Control de Aduanas. 2013. U.S. Immigration and Customs Enforcement, ICE. http://www.ice.gov/best/ (5 de junio de 2014).

Sistema Nacional de Seguridad Pública. 2013. Cifras de la incidencia al delito del Secretariado Ejecutivo del Sistema Nacional de Seguridad Pública. http://secretariadoejecutivo.gob.mx/es/SecretariadoEjecutivo/09082013 (10 de junio de 2014).

Tenenhaus, Michel. 2008. Component-based structural equation modelling. Total Quality Management and Business Excellence 19 (7/8): 871-886.

Tenenhaus, Michel. 2005. Structural equation modelling for small samples. file:///C:/Users/Dell/Downloads/CR885Tenenhaus. pdf (5 de junio de 2014).

Vega-Briones, Germán y Giovanna Román-Robayo. 2010. Migración y diversas expresiones de violencia en la frontera norte de México y Colombia. Simposio 5: Conexiones y desconexiones. La criminalización de la migración internacional. http://www.tratacongreso2010.org/docs/Germ\%C3\%A1n\%20Vega\%20y\%20 Giovanna\%20Rom\%C3\%A1n.pdf (12 de mayo de 2014).

Woolcock, Michael y Deepa Narayan. 1997. Capital social: implicaciones para la teoría, la investigación y las políticas sobre desarrollo. http://preval.org/documentos/00418.pdf (10 de enero de 2014). 